\title{
Osseous Pseudoprogression in Vertebral Bodies Treated with Stereotactic Radiosurgery: A Secondary Analysis of Prospective Phase I/II Clinical Trials
}

\author{
(D) B. Amini, (D) C.B. Beaman, (D).E. Madewell, (DP.K. Allen, (D).D. Rhines, DC.E. Tatsui, (DN.M. Tannir, (D). Li, (DP.D. Brown,
} and (1D)A.J. Ghia

\begin{abstract}
BACKGROUND AND PURPOSE: Osseous pseudoprogression on MR imaging can mimic true progression in lesions treated with spine stereotactic radiosurgery. Our aim was to describe the prevalence and time course of osseous pseudoprogression to assist radiologists in the assessment of patients after spine stereotactic radiosurgery.
\end{abstract}

MATERIALS AND METHODS: A secondary analysis of 2 prospective trials was performed. MRIs before and after spine stereotactic radiosurgery were assessed for response. "Osseous pseudoprogression" was defined as transient growth in signal abnormality centered at the lesion with a sustained decline on follow-up MR imaging that was not attributable to chemotherapy.

RESULTS: From the initial set of 223 patients, 37 lesions in 36 patients met the inclusion criteria and were selected for secondary analysis. Five of the 37 lesions (14\%) demonstrated osseous pseudoprogression, and 9 demonstrated progressive disease. There was a significant association between single-fraction therapy and the development of osseous pseudoprogression $(P=.01)$, and there was a significant difference in osseous pseudoprogression-free survival between single- and multifraction regimens $(P=.005)$. In lesions demonstrating osseous pseudoprogression, time-to-peak size occurred between 9.7 and 24.4 weeks after spine stereotactic radiosurgery (mean, 13.9 weeks; $95 \% \mathrm{Cl}, 8.6-19.1$ weeks). The peak lesion size was between 4 and $10 \mathrm{~mm}$ larger than baseline. Most lesions returned to baseline size between 23 and 52.4 weeks following spine stereotactic radiosurgery.

CONCLUSIONS: Progression on MR imaging performed between 3 and 6 months following spine stereotactic radiosurgery should be treated with caution because osseous pseudoprogression may be seen in more than one-third of these lesions. Single-fraction spine stereotactic radiosurgery may be associated with osseous pseudoprogression. The possibility of osseous pseudoprogression should be incorporated into the prospective criteria for assessment of local control following spine stereotactic radiosurgery.

ABBREVIATIONS: OPP = osseous pseudoprogression; $\mathrm{PD}$ = progressive disease; $\mathrm{PR}$ = partial response; $\mathrm{SSRS}$ = spine stereotactic radiosurgery

S pine stereotactic radiosurgery (SSRS) is a form of stereotactic body radiation therapy that combines advanced treatment delivery techniques (eg, intensity-modulated radiation therapy) with image guidance and rigid immobilization to deliver high doses of conformal radiation to the target while minimizing

Received June 14, 2015; accepted after revision July 14.

From the Departments of Diagnostic Radiology (B.A., J.E.M.), Biostatistics (P.K.A.), Neurosurgery (L.D.R., C.E.T.), Genitourinary Medical Oncology (N.M.T.), and Radiation Oncology (J.L., P.D.B., A.J.G.), The University of Texas MD Anderson Cancer Center, Houston, Texas; and The University of Texas Health Science Center at Houston (C.B.B.), Houston, Texas.

This work was supported in part by the Cancer Center Support Grant (National Cancer Institute Grant P30 CA016672).

Please address correspondence to Behrang Amini, MD, PhD, Department of Diagnostic Radiology, University of Texas MD Anderson Cancer Center, 1400 Pressler St, Unit 1475, Houston, TX 77030; e-mail: bamini@mdanderson.org; @DrBehrangAmini

-- Indicates open access to non-subscribers at www.ajnr.org

http://dx.doi.org/10.3174/ajnr.A4528 exposure to nearby critical structures such as the spinal cord (Fig $1 A-C$ ). Imaging plays a critical role not only in diagnosis and therapy planning but also in the post-SSRS evaluation of patients in determining the response to therapy. In evaluating patients following SSRS, we have noticed a phenomenon whereby the intraosseous extent of the lesion transiently enlarges on early posttherapy MRI and simulates progression (Fig 1D-F).

This osseous pseudoprogression (OPP) complicates the early post-SSRS imaging assessment of patients and can lead to uncertainty about the true response. We undertook a secondary analysis of 2 prospective trials performed at our institution to define the prevalence and time course of OPP to provide guidance to interpreting radiologists in assessing the patient after SSRS.

\section{MATERIALS AND METHODS Patient Population}

We performed a secondary analysis of 2 consecutive prospective SSRS trials at our institution. Between 2002 and 2011,223 patients 


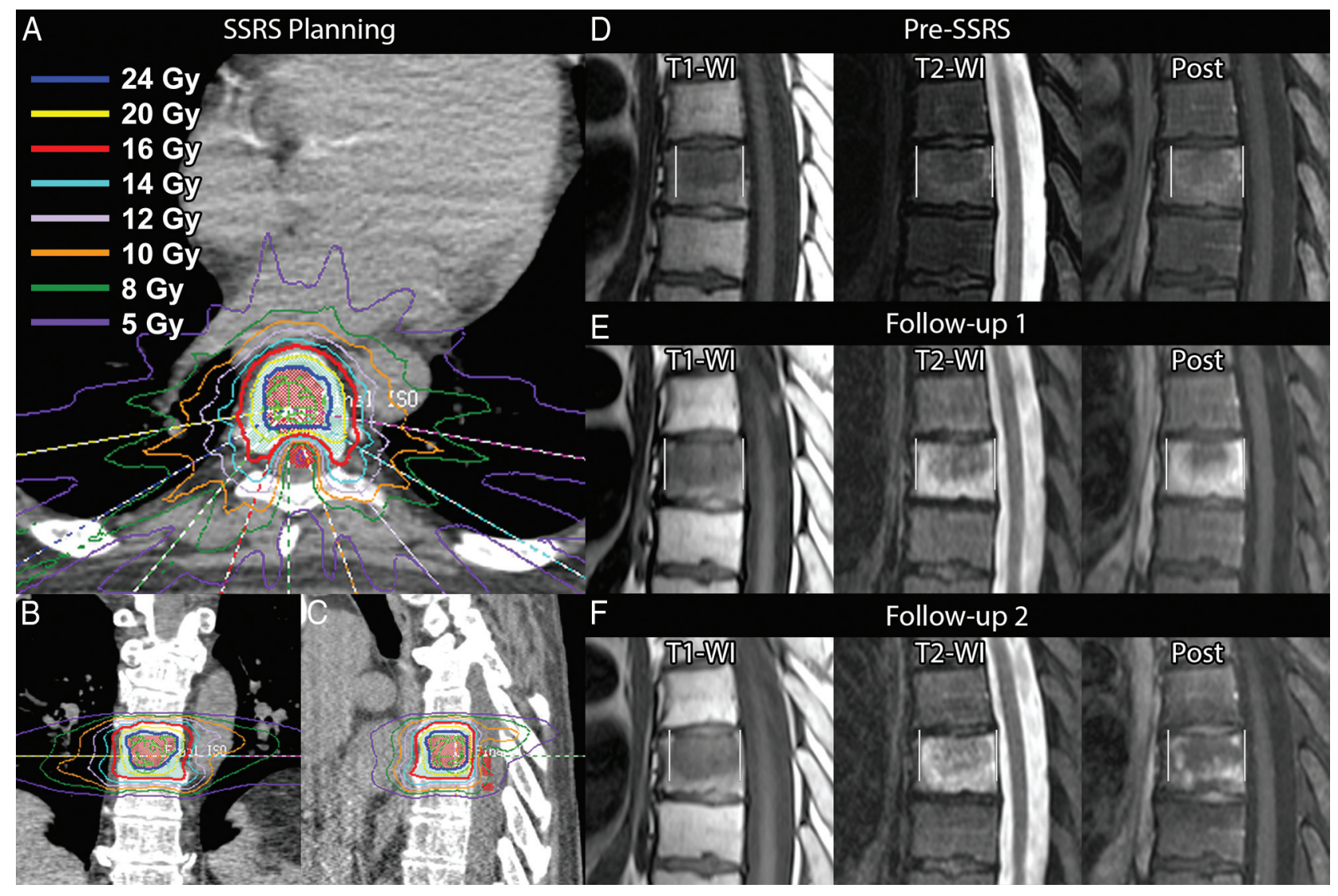

FIG 1. Index case of osseous pseudoprogression (not part of the analysis). A 55-year-old man with metastatic renal cell carcinoma. A-C, Axial, coronal, and sagittal images from the SSRS treatment plan. Twenty-four Gy (blue open area) will be delivered to the gross tumor volume corresponding to the metastasis seen on MR imaging $(D)$, and $16 \mathrm{~Gy}$ (red open area), to the remainder of the at-risk vertebral body (clinical tumor volume) in a single fraction. The spinal cord (red shaded area) is spared from high-dose radiation. Other colors correspond to isodose lines as indicated. D, MR image obtained 6 weeks before therapy shows the lesion in the central vertebral body. E, The lesion enlarges on follow-up 1 , 6 weeks after SSRS. There is extensive signal abnormality surrounding the lesion. The entire extent of the signal abnormality, including the lesion, is measured in this study (indicated by vertical white lines). F, Surrounding signal abnormality decreases on follow-up 2, 16 weeks following SSRS.

were enrolled in 2 phase I/II trials at our institution, evaluating the use of single-fraction and multiple-fraction SSRS, respectively, in patients with spinal metastases. All patients treated in the initial study received multifraction SSRS. As the safety of multifraction SSRS was established, a second study was initiated in which patients were treated with single-fraction SSRS. The trials were approved by the institutional review board, and written informed consent was obtained from trial participants before enrollment. Eligibility requirements included diagnosis of cancer, Karnofsky Performance Status score of $>40$, and MR imaging identifying spinal or paraspinal metastasis within 4 weeks of enrollment. Indications for treatment included oligometastatic disease from a known primary tumor, failure of prior surgery or conventionally fractionated radiation, residual tumor after surgery, medical inoperability, and refusal of surgery. Exclusion criteria included spinal cord compression, unstable spine as determined by a multidisciplinary tumor board, cytotoxic chemotherapy within 1 month of enrollment, and external beam radiation therapy to the current site of disease 3 months before planned SSRS.

Exclusion criteria for the secondary analysis included the following: 1) imaging follow-up of $<6$ months, 2) the presence of surgical hardware or cement augmentation during the observation period, 3 ) the presence of significant fracture during the ob- servation period ("significant fracture" defined as one that results in signal abnormality that could not be differentiated from the lesion), 4) lesions not located in the vertebral body (to allow reproducible measurements), and 5) lesions occupying $>75 \%$ of the vertebral body cross-sectional area (to allow determination of lesion growth).

\section{Radiation Therapy Procedure}

All patients underwent intensity-modulated, near-simultaneous, CT-guided SSRS by using the EXaCT Targeting system CT-onrails (GE Healthcare, Milwaukee, Wisconsin) or the Trilogy treatment delivery system with On-Board Imager Conebeam CT (Varian Medical Systems, Palo Alto, California), as previously described. ${ }^{1,2}$ Patients were immobilized in a BodyFix 14 stereotactic body frame system (Elekta, Stockholm, Sweden) and aligned by using a BodyFix 14 Target Positioner (Elekta). Treatment planning was performed by using intensity-modulated radiation therapy inverse-treatment planning software (Pinnacle; Philips Medical Systems, Andover, Massachusetts). Verification of target positioning and quality assurance procedures for each case was performed by the radiation oncologist and a dedicated radiation physicist, respectively.

Spinal tumors were prescribed to receive 30 Gy in 5 fractions, 
27 Gy in 3 fractions, or 16, 18, or 24 Gy in 1 fraction to the gross tumor volume. "Gross tumor volume" was defined by the visible disease on MR imaging fused with the pretreatment planning CT. "Clinical treatment volume" was defined as gross tumor volume with at-risk contiguous bone marrow. "Planning treatment volume" was defined with no margin to the clinical treatment volume. In most patients, $80 \%-90 \%$ of the target volume received the prescription dose. For the single-fraction protocol, the maximal point dose to $0.01 \mathrm{~cm}^{3}$ of the spinal cord was $10 \mathrm{~Gy}$ based on dose-volume histogram analysis. For the multifraction protocol, the spinal cord constraint was maximum dose to $10 \mathrm{~Gy}$ for 5 -fraction treatments and 9 Gy for the 3-fraction treatments. Radiation treatments were administered on alternating days.

\section{Image Review and Analysis}

Patients were seen for follow-up visits and MR imaging every 3 months for 2 years, then every 6 months thereafter. MR imaging was performed in most cases without and with intravenous gadolinium-based contrast material, with exceptions made for compromised renal function (glomerular filtration rate of $<30 \mathrm{~mL}$ / $\mathrm{min} / 1.73 \mathrm{~m}^{2}$ ) and patient refusal. The screening review of clinical records and MR images for application of the exclusion criteria (see "Patient Population") was performed by a single reader (C.B.B.). Lesions were measured in consensus by 2 readers (C.B.B. and B.A.) on sagittal T1WI, T2WI fat suppressed, and postcontrast sequences as the greatest anteroposterior extent of signal abnormality (Fig 1E, $-F$ ). Because the aim of the study was to define the phenomenon of osseous pseudoprogression, emphasis was placed on the reproducibility of measurements. Axial images were not used for measurement due to scan-to-scan variation related to obliquity.

Lesion response was determined retrospectively by comparing the post-SSRS MRIs with the pre-SSRS baseline study as a partial response ( $\mathrm{PR}$, decrease in size of the lesion of $>2 \mathrm{~mm}$ ), stable disease (no growth or growth of $<2 \mathrm{~mm}$ ), progressive disease ( $\mathrm{PD}$, continued growth of $>2 \mathrm{~mm}$ over multiple studies), and osseous pseudoprogression. "OPP" was defined as transient growth of $>2 \mathrm{~mm}$ in signal abnormality centered at the lesion that was followed by sustained decline ( $\pm 2 \mathrm{~mm}$ in $>3$ months) in the size of the area of signal abnormality. The medication history and the response of other bone metastases (if present) were reviewed to ensure that this decline was not due to systemic therapy by checking that no change in systemic therapy occurred following peak lesion size. If systemic therapy was changed following peak lesion size, a determination of OPP was made if other bone lesions enlarged while the SSRS-treated lesion decreased in size (ie, the decrease in size of the SSRS-treated lesion could not be attributed to the change in systemic therapy). If an enlarging lesion failed to meet at least 1 of the above criteria, it was classified as PD. Two millimeters was chosen as the measurement error based on the spatial resolution of MR imaging.

\section{Statistical Analysis}

Statistical analysis was performed by using R statistical computing software for Windows (http://www.r-project.org) and STATA/MP 14.0 (StataCorp, College Station, Texas). Univariate analysis was performed by using Cox proportional hazards regres- sion modeling with OPP-free survival as the evaluable end point. The Fisher exact test was used to assess the association between the OPP and non-OPP groups with respect to fractionation regimens (single or multiple). Tumor histology was dichotomized as radiosensitive and radioresistant (ie, sensitive or resistant to conventional radiation therapy), with the latter group consisting of patients with renal cell carcinoma, sarcoma, adenoid cystic carcinoma, and melanoma. Kaplan-Meier analysis and the log-rank test were used for assessment of OPP-free survival. Statistical significance was set for $P$ values $<.05$.

\section{RESULTS}

From the initial prospective dataset of 223 patients, 37 lesions in 36 patients met the inclusion criteria and were selected for secondary analysis. Demographic and clinical data are presented in Table 1. The mean age at SSRS was 53 years (range, 32-74 years; 95\% CI, 49-56 years). Metastases from renal cell carcinoma, sarcoma, and thyroid carcinoma constituted $67 \%$ of cases. Most treated lesions were in the thoracic and lumbar spine $(49 \%$ and $43 \%$, respectively).

Sixteen lesions $(43 \%)$ received single-fraction therapy, 15 $(41 \%)$ received 3 fractions, and $6(16 \%)$ received 5 fractions. The most common dose/fraction combination was $27 \mathrm{~Gy}$ at 3 fractions (41\%), followed by 24 Gy at 1 fraction (19\%), 18 Gy at 1 fraction, 30 Gy at 5 fractions (16\% each), and 16 Gy at 1 fraction (8\%).

Most lesions (62\%) either stabilized or partially responded to therapy during the observation period. The remaining 14 lesions (38\%) enlarged on the early post-SSRS scans. Five of these (36\%) demonstrated OPP, while 9 (64\%) demonstrated progressive disease.

The overall prevalence of OPP among all 37 lesions was 14\%. The 5 cases of OPP all received single-fraction SSRS, resulting in a prevalence of $31 \%$ when limited to a population of lesions treated with single-fraction radiation therapy. Three of these 5 received $24 \mathrm{~Gy}$, with the remaining 2 treated with 16 and 18 Gy each.

Univariate analysis (Table 2) revealed an association between patient age and the development of OPP (hazard ratio, 1.16; 95\% CI, 1.03-1.30). Linear regression failed for the fractionation regimen (single- versus multifraction) due to collinearity (all cases of OPP occurred in lesions treated with single-fraction therapy). The Fisher exact test found a significant association between singlefraction therapy and the development of $\mathrm{OPP}(P=.01)$, and there was a significant difference in OPP-free survival between singleand multifraction regimens $(P=.005$, Fig 2$)$.

No significant association was found on univariate analysis for the development of OPP with respect to tumor histology (radioresistant versus radiosensitive), dose, or location in the spine (thoracic versus cervical/lumbar).

The apparent change in size of the lesions after SSRS is shown in Fig 3. The time-to-peak lesion size occurred between 9.7 and 24.4 weeks after SSRS (mean, 13.9 weeks; 95\% CI, 8.6-19.1 weeks). The mean change in size from the baseline preradiation MR imaging to peak size was $5 \mathrm{~mm}$ on T1WI (range, $4-8 \mathrm{~mm}$ ), 5 $\mathrm{mm}$ on T2WI fat suppressed (range, 2-10 $\mathrm{mm}$ ), and $6 \mathrm{~mm}$ on postcontrast images (range, $4-9 \mathrm{~mm}$ ), with no significant difference among the 3 .

The lesions returned to baseline size (within an MR imaging

AJNR Am J Neuroradiol 37:387-92 Feb 2016 www.ajnr.org 
Table 1: Demographic and clinical data

\begin{tabular}{|c|c|c|c|c|c|c|c|}
\hline No. & Age (yr) & Sex & Histology & Level & Dose (Gy) & Fractions & Response \\
\hline 1 & 57 & $\mathrm{~F}$ & Renal cell carcinoma & $\mathrm{Ll}$ & 24 & 1 & OPP \\
\hline 3 & 55 & $M$ & Renal cell carcinoma & L4 & 24 & 1 & SD \\
\hline 4 & 54 & $\mathrm{~F}$ & Renal cell carcinoma & $\mathrm{T} 12$ & 24 & 1 & PD \\
\hline 5 & 52 & $M$ & Renal cell carcinoma & L1 & 27 & 3 & PR \\
\hline 8 & 52 & $M$ & Renal cell carcinoma & L4 & 30 & 5 & PD \\
\hline 9 & 42 & $M$ & Renal cell carcinoma & T11 & 30 & 5 & SD \\
\hline 11 & 53 & $\mathrm{~F}$ & Renal cell carcinoma & $\mathrm{T} 12$ & 30 & 5 & PR \\
\hline 12 & 60 & $\mathrm{~F}$ & Breast carcinoma & T8 & 30 & 5 & SD \\
\hline 13 & 63 & $\mathrm{~F}$ & Melanoma & L1 & 27 & 3 & SD \\
\hline 15 & 58 & $M$ & Basal cell carcinoma & $\mathrm{T} 4$ & 30 & 5 & PD \\
\hline 16 & 32 & $\mathrm{~F}$ & Thyroid carcinoma & L3 & 30 & 5 & SD \\
\hline \multirow[t]{2}{*}{17} & 46 & $\mathrm{~F}$ & Thyroid carcinoma & $\mathrm{L} 1$ & 27 & 3 & PR \\
\hline & & & & L2 & 27 & 3 & SD \\
\hline 18 & 50 & $\mathrm{~F}$ & Thyroid carcinoma & T11 & 27 & 3 & SD \\
\hline 19 & 49 & $M$ & Thyroid carcinoma & T6 & 27 & 3 & PD \\
\hline 20 & 44 & $\mathrm{~F}$ & Sarcoma & $\mathrm{C} 3$ & 27 & 3 & PR \\
\hline 22 & 50 & $\mathrm{~F}$ & Sarcoma & T8 & 27 & 3 & SD \\
\hline 23 & 70 & $\mathrm{~F}$ & Sarcoma & L2 & 27 & 3 & PR \\
\hline 24 & 49 & $M$ & Thyroid carcinoma & $\mathrm{T1}$ & 27 & 3 & SD \\
\hline 25 & 48 & $\mathrm{~F}$ & Breast carcinoma & $\mathrm{T} 2$ & 18 & 1 & PR \\
\hline 26 & 51 & $\mathrm{~F}$ & Breast carcinoma & T5 & 18 & 1 & SD \\
\hline 27 & 38 & $\mathrm{~F}$ & Lung carcinoma & L1 & 18 & 1 & $\mathrm{SD}$ \\
\hline 29 & 74 & $M$ & Lung carcinoma & L1 & 18 & 1 & OPP \\
\hline 30 & 35 & $M$ & Sarcoma & T8 & 16 & 1 & PR \\
\hline 31 & 50 & $\mathrm{~F}$ & Sarcoma & T5 & 18 & 1 & PD \\
\hline 32 & 59 & $M$ & Sarcoma & $\mathrm{T} 5$ & 18 & 1 & SD \\
\hline 35 & 44 & $M$ & Renal cell carcinoma & L4 & 24 & 1 & PD \\
\hline 36 & 63 & $M$ & Renal cell carcinoma & L6 & 24 & 1 & OPP \\
\hline 37 & 70 & $M$ & Renal cell carcinoma & $\mathrm{T12}$ & 24 & 1 & OPP \\
\hline 38 & 65 & $M$ & Renal cell carcinoma & $\mathrm{C} 3$ & 24 & 1 & PR \\
\hline 39 & 61 & $M$ & Renal cell carcinoma & T6 & 27 & 3 & PD \\
\hline 40 & 33 & $\mathrm{~F}$ & Breast carcinoma & $\mathrm{L} 4$ & 27 & 3 & PD \\
\hline 41 & 67 & $M$ & Colon carcinoma & L3 & 27 & 3 & PR \\
\hline 42 & 48 & $\mathrm{~F}$ & Adenoid cystic carcinoma & $\mathrm{C} 2$ & 27 & 3 & PD \\
\hline 43 & 58 & $\mathrm{~F}$ & Thyroid carcinoma & L2 & 27 & 3 & PR \\
\hline 44 & 34 & $\mathrm{~F}$ & Breast carcinoma & T9 & 16 & 1 & PR \\
\hline 45 & 65 & $M$ & Prostate carcinoma & T6 & 16 & 1 & OPP \\
\hline
\end{tabular}

Note:-SD indicates stable disease.

Table 2: Univariate Cox regression analysis for OPP-free survival

\begin{tabular}{lcccl}
\hline \multicolumn{1}{c}{ Variable } & HR & $\boldsymbol{P}$ Value & $\mathbf{9 5 \% ~ C l}$ & \multicolumn{1}{c}{ Comment } \\
\hline Age & 1.16 & .01 & $1.03-1.30$ & Continuous \\
Radiation dose & 0.86 & .1 & $0.72-1.03$ & Continuous \\
Tumor radiosensitivity & 0.70 & .7 & $0.12-4.17$ & Radiosensitive vs -resistant \\
Location & 0.75 & .8 & $0.13-4.48$ & T-spine vs C/L-spine \\
Fraction & - & - & - & Collinearity (all OPP occurred in \\
& & & & single-fraction regimens) \\
\hline
\end{tabular}

Note:- HR indicates hazard ratio; C/L, cervical/lumbar; -, not applicable.

measurement error of $\pm 2 \mathrm{~mm}$ ) on T1WI after 23.1-52.4 weeks (mean, 32 weeks). One lesion each on T2WI and postcontrast images did not return to baseline size within the observation period. The mean time to return to baseline size, excluding these lesions, was 30.6 weeks for T2WI (range, 23-37.4 weeks) and 35 weeks for postcontrast images (range, 25.3-52.4 weeks).

\section{DISCUSSION}

The phenomenon of pseudoprogression has been classically described in brain lesions following gamma knife therapy. ${ }^{3}$ With expanded use of stereotactic body radiation therapy, pseudoprogression has been described and analyzed in lung lesions ${ }^{4,5}$ and more recently reported in the soft-tissue components of 2 bone lesions following SSRS. ${ }^{6}$ However, no systematic analysis or even anecdotal reports of pseudoprogression of lesions confined to bone have been published, to our knowledge.

The absence of these data complicates the follow-up assessment of patients treated with SSRS and can lead to uncertainty regarding the true response to radiation in the setting of clinical trials. Our study provides preliminary information on the prevalence, time course, and risk factors for the development of OPP and can provide guidance on the management of enlarging lesions on early post-SSRS scans.

We found that OPP is common, with a prevalence of $14 \%$ overall and $31 \%$ when considering its occurrence in the population of patients treated with single-fraction SSRS. All 5 cases of OPP in our study occurred after single-fraction SSRS (24 Gy, 18 Gy, and 16 Gy). A significant association was present between single-fraction therapy and OPP, and there was a significant difference in OPP-free survival between single- and multifraction regimens $(P=.005$, Fig $2)$. Most interesting, the 2 recently reported cases of pseudoprogression of soft-tissue components of bone lesions have also been in single-fraction regimens. ${ }^{6}$ This finding suggests that the higher biologic dose delivered by singlefraction regimens plays a role in the development of OPP.

The etiology of OPP is likely related to a combination of the effect of radiation on the tumor itself and the adjacent marrow. Tumor growth due to necrosis in response to therapy (chemotherapy and radiation) is a well-known phenomenon, ${ }^{7}$ which likely plays a role in the development of OPP. Indeed, a recent case report in a patient with pseudoprogression in the soft-tissue (epidural) component of a spine lesion following SSRS showed necrosis in the early ( $<8$ weeks) post-SSRS period. ${ }^{6}$ The effect of radiation on the surrounding bone can be extrapolated from experimental data in animals on the effect of conventional radiation therapy with time and compared with the time course of OPP (Fig 3). In the range of radiation doses used in our study (16-24 Gy), manifestations of radiation on the bone marrow during the first 4 weeks included progressive decrease in marrow cellularity, decrease in the number of sinusoids, and increase in endosteal fibrosis. ${ }^{8}$ This sequence of events was followed by a marked decrease in hematopoietic activity and a marked disruption of sinusoids, with free flow of erythrocytes into parenchymal areas of the marrow. ${ }^{8}$ Hematopoietic cellularity, which normally accounts for the T2 signal 
of marrow, was relatively suppressed at this time; however, the extravasation of blood elements through disrupted sinusoids can account for the increase in marrow T2 signal, and the increased permeability of the sinusoids can account for the increased enhancement during the peak of OPP. Later, there is further suppression of marrow hematopoiesis, disappearance of sinusoidal elements, and maximal endosteal fibrosis. ${ }^{8}$ This relatively acellular phase can account for the decrease in marrow T2 signal and enhancement seen on the downslope of the OPP curve. Following this period, there is patchy early regeneration of marrow and sinusoid-like structures, which progress to an irregular formation of sinusoids within areas of hematopoiesis ${ }^{8}$

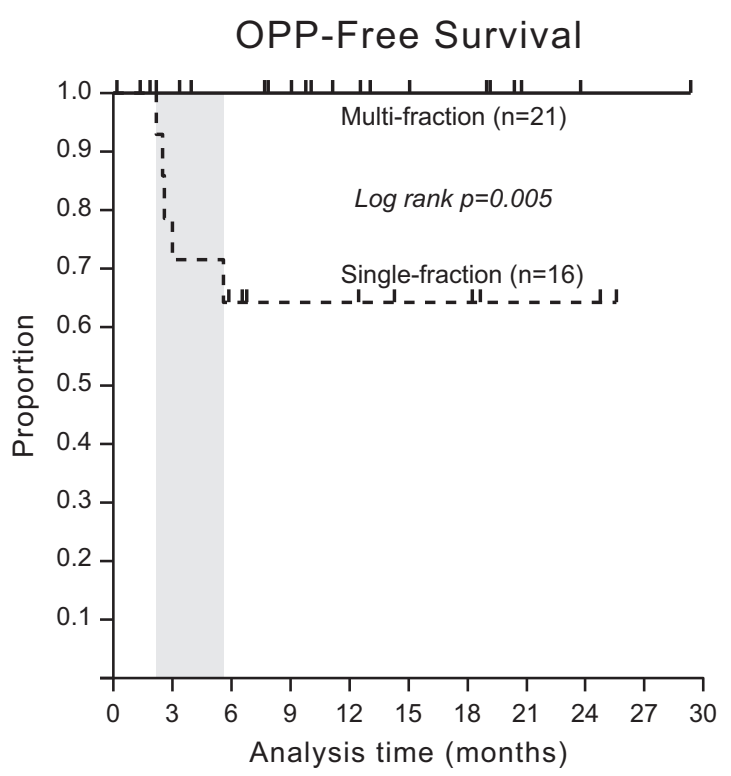

FIG 2. Osseous pseudoprogression-free survival by SSRS fraction status. OPP-free survival is defined as the length of time following SSRS that a patient lives with the disease without development of OPP. Kaplan-Meier analysis shows that there was a significant difference in OPP-free survival between single- and multifraction regimens. The 5 cases of OPP were all treated with single-fraction SSRS and occurred between approximately 3 and 6 months following SSRS (shaded area).
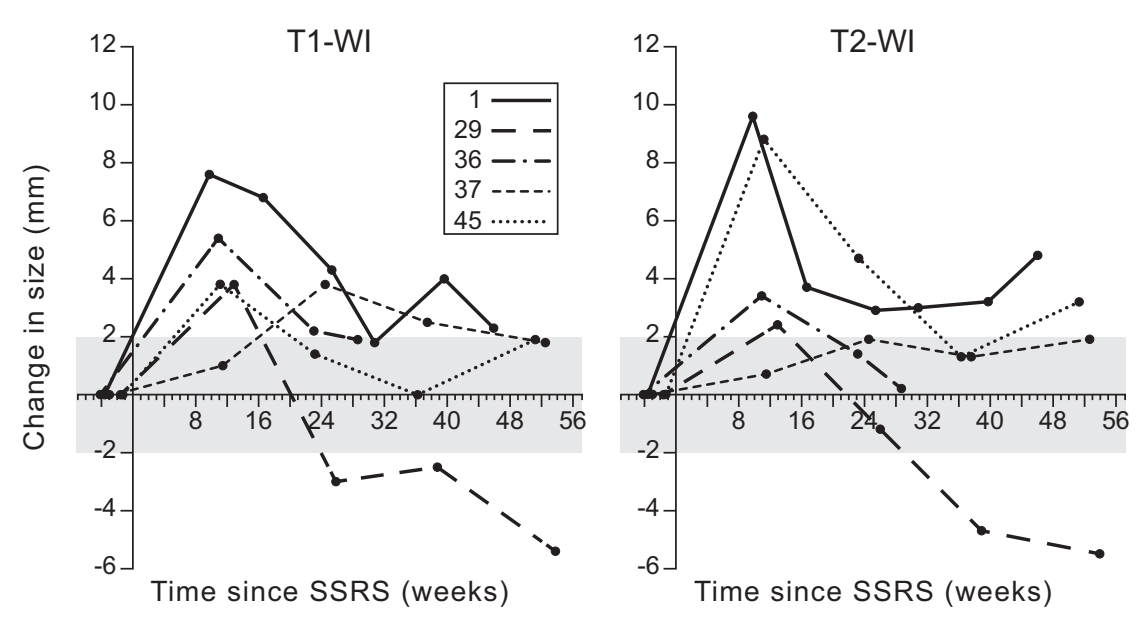

and may account for the progressive normalization of marrow signal following the early downslope.

The time-to-peak size in OPP occurred between 9.7 and 24.4 weeks following SSRS and returned to baseline between 23 and 52.4 weeks after SSRS, with slight variation depending on the pulse sequence (Fig 3). This range is earlier than that of the reported time-to-peak lesion size (24-48 weeks) and resolution (60-96 weeks) for early pseudoprogression of brain lesions following gamma knife therapy. ${ }^{9,10}$ This difference is not unexpected, given the differences between the local environments encountered in bone and brain. Temporal evolution information was not obtained in the aforementioned case series on soft-tissue pseudoprogression ${ }^{6}$ because both reported lesions were treated following the development of pseudoprogression due to symptoms (steroids in one case and laminectomy in the other).

The aspect of this study that is most relevant to clinical practice is the guidance it can provide to radiologists on the interpretation of an enlarging lesion on early post-SSRS scans. As noted above, of the 14 lesions in our study that enlarged on early post-SSRS MRI, $5(36 \%)$ represented OPP. The impact of this finding on clinical practice is that enlarging lesions on early post-SSRS scans will require follow-up imaging to elucidate the true response because more than one-third would be expected to have OPP and not true PD.

Our study has several limitations. While our cases were derived from 2 prospective datasets, the data were not randomized and biases may exist between the 2 primary cohorts of patients. A related limitation of this secondary retrospective analysis is the necessity of excluding 187 of the original 223 patients due to a combination of factors that would have complicated detection of OPP (eg, hardware placement, cement augmentation, and moderate to severe pathologic fracture). A larger validation study will be needed before the results of this preliminary study can be confidently generalized.

Another limitation of our study is that many patients were receiving systemic therapy during the observation period. Our retrospective response criteria attempted to account for the effect of systemic therapy by considering OPP when no change in sys-

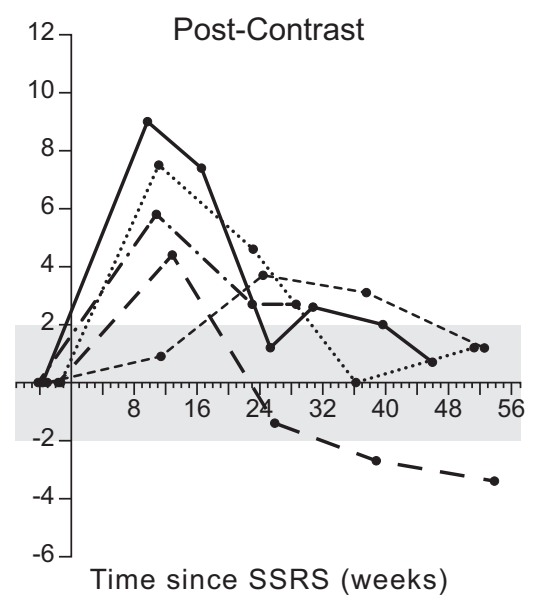

FIG 3. Time course of lesions demonstrating osseous pseudoprogression. Lesions 1, 29, 36, 37, and 45 (see Table 1) demonstrate an initial increase in size followed by a decrease (OPP). The shaded areas correspond to a \pm 2 - $\mathrm{mm}$ change from baseline, representing measurement error on MR imaging. Similarly, the apparent late increase in lesion size on T2WI in patients 1, 37, and 45 falls within a \pm 2 -mm range of the preceding time points and may represent measurement error. 
temic therapy had occurred following peak lesion size or, if systemic therapy was changed following peak lesion size, by considering OPP when the response of the treated lesion could be shown to be different from that of other bone lesions (ie, the SSRStreated lesion decreased in size, while other bone lesions enlarged). However, the effect of synergy between radiation and systemic therapy cannot be entirely eliminated by applying these criteria to a retrospective dataset.

A limitation inherent in our study design, which aimed to describe the time course and prevalence of a new phenomenon, is that it limits comment on imaging features that would allow a prospective differentiation of OPP from PD. A separate, multireader study with test and validation arms would be needed to establish criteria for differentiating the 2 . However, in the case of pseudoprogression in the brain, findings on conventional MR imaging have been insufficient in making this distinction, ${ }^{11}$ and advanced imaging techniques such as diffusion and perfusion MR imaging have been investigated for this purpose. ${ }^{12}$

A final limitation relates to the retrospective determination of response based on the change in lesion size with time. While we believe that it is reasonable to assume that persistently enlarging lesions represented PD and transiently enlarging lesions represented OPP, no pathologic proof was available in our patients.

Despite these limitations, our study is the first to describe and systematically analyze the prevalence and time course of OPP following SSRS and can provide guidance in the assessment of the patient after SSRS.

\section{CONCLUSIONS}

Progression on MR imaging performed between 3 and 6 months following SSRS should be treated with caution because an osseous pseudoprogression may be seen in more than one-third of these lesions. Serial MR imaging is needed to determine the true response. The possibility of OPP should be incorporated into prospective criteria for the assessment of local control following SSRS.

Disclosures: Charles B. Beaman—UNRELATED: Grants/Grants Pending: Vision Training Grant, Comments: pays my tuition and salary of approximately $\$ 21,000$ per year. Laurence D. Rhines-UNRELATED: Consultancy: Stryker, Globus medical, Comments: Educational.

\section{REFERENCES}

1. Chang EL, Shiu AS, Lii MF, et al. Phase I clinical evaluation of nearsimultaneous computed tomographic image-guided stereotactic body radiotherapy for spinal metastases. Int J Radiat Oncol Biol Phys 2004;59:1288-94 CrossRef Medline

2. Shiu AS, Chang EL, Ye JS, et al. Near simultaneous computed tomography image-guided stereotactic spinal radiotherapy: an emerging paradigm for achieving true stereotaxy. Int J Radiat Oncol Biol Phys 2003;57:605-13 CrossRef Medline

3. de Wit MC, de Bruin HG, Eijkenboom W, et al. Immediate postradiotherapy changes in malignant glioma can mimic tumor progression. Neurology 2004;63:535-37 CrossRef Medline

4. Huang K, Dahele M, Senan S, et al. Radiographic changes after lung stereotactic ablative radiotherapy (SABR): can we distinguish recurrence from fibrosis? A systematic review of the literature. $\mathrm{Ra}$ diother Oncol 2012;102:335-42 CrossRef Medline

5. Dahele M, Palma D, Lagerwaard F, et al. Radiological changes after stereotactic radiotherapy for stage I lung cancer. J Thorac Oncol 2011;6:1221-28 CrossRef Medline

6. Taylor DR, Weaver JA. Tumor pseudoprogression of spinal metastasis after radiosurgery: a novel concept and case reports. J Neurosurg Spine 2015;22:534-39 CrossRef Medline

7. Choi H, Charnsangavej C, Faria SC, et al. Correlation of computed tomography and positron emission tomography in patients with metastatic gastrointestinal stromal tumor treated at a single institution with imatinib mesylate: proposal of new computed tomography response criteria. J Clin Oncol 2007;25:1753-59 CrossRef Medline

8. Knospe WH, Blom J, Crosby WH. Regeneration of locally irradiated bone marrow, I: dose dependent, long-term changes in the rat, with particular emphasis upon vascular and stromal reaction. Blood 1966;28:398-415 Medline

9. Hayhurst $\mathrm{C}$, Zadeh G. Tumor pseudoprogression following radiosurgery for vestibular schwannoma. Neuro Oncol 2012;14:87-92 CrossRef Medline

10. Bakardjiev AI, Barnes PD, Goumnerova LC, et al. Magnetic resonance imaging changes after stereotactic radiation therapy for childhood low grade astrocytoma. Cancer 1996;78:864-73 CrossRef Medline

11. Young RJ, Gupta A, Shah AD, et al. Potential utility of conventional MRI signs in diagnosing pseudoprogression in glioblastoma. $\mathrm{Neu}$ rology 2011;76:1918-24 CrossRef Medline

12. Prager AJ, Martinez N, Beal K, et al. Diffusion and perfusion MRI to differentiate treatment-related changes including pseudoprogression from recurrent tumors in high-grade gliomas with histopathologic evidence. AJNR Am J Neuroradiol 2015;36:877-85 CrossRef Medline 